09

\title{
Получение изолированных аттосекундных импульсов с большой электрической площадью в плотной резонансной среде
}

\author{
(C) Р.М. Архипов ${ }^{1,2}$, М.В. Архипов ${ }^{1}$, С.В. Федоров ${ }^{2}$, Н.Н. Розанов ${ }^{2}$ \\ ${ }^{1}$ Санкт-Петербургский государственный университет, \\ 199034 Санкт-Петербург, Россия \\ ${ }^{2}$ ФТИ им. А.Ф. Иоффре РАН, \\ 194021 Санкт-Петербург, Россия \\ e-mail: arkhipovrostislav@gmail.com, m.arkhipov@spbu.ru, sfedorov2006@bk.ru, nnrosanov@mail.ru
}

Поступила в редакцию 28.06.2021 г.

В окончательной редакции 28.06.2021 г.

Принята к публикации 10.07.2021 г.

Показано, что в резонансной среде малоцикловый импульс может быть превращен в два униполярных импульса, разнесенных во времени на расстояние, на порядок и более превышающее длительность исходного импульса. Это позволяет в ряде задач рассматривать такие импульсы по отдельности как униполярные. Проведена оценка величины электрической площади относительно ее „атомной меры“.

Ключевые слова: аттосекундные импульсы, униполярные импульсы, субцикловые импульсы, электрическая площадь импульсов.

DOI: $10.21883 /$ OS.2021.10.51495.2512-21

\section{Введение}

Импульсы в аттосекундном диапазоне длительностей содержат несколько циклов колебаний, что позволяет использовать их для изучения сверхбыстрой динамики волновых пакетов в атомах, молекулах и твердых телах [1-6]. В них компоненты вектора напряженности поля несколько раз меняют направление на противоположное за время длительности импульса. Поэтому электрическая площадь таких импульсов, вычисляемая как интеграл от напряженности электрического поля по времени $S_{E}=\int E(t) d t$, равна 0 , и они не являются униполярными [7].

Сократить длительность импульса, содержащего один цикл колебаний, можно, если „обрезать“ одну из полуволн поля и превратить его в униполярный полуцикловый импульс, содержащий полуволну поля одной полярности.

Униполярные импульсы за счет нерезонансного воздействия на микрообъекты способны быстро и более эффективно изменять состояния квантовых систем по сравнению с длинными многоцикловыми импульсами $[3,4,8-$ 14], ускорять заряженные частицы [15], поворачивать спин электрона $[16,17]$, осуществлять голографическую запись со сверхвысоким временным разрешением [18].

Для униполярных импульсов одной из важнейших характеристик является электрическая площадь импульса $S_{E}$. Воздействие таких импульсов на микробъекты определяется именно электрической площадью импульса, а не его энергией [9-17].

В экспериментах и в теоретических исследованиях чаще всего удается получить квазиуниполярные импульсы, содержащие однополярный всплеск поля с большой амплитудой и длинный задний фронт противоположной полярности [3,5,19-23]. Как показывают теоретические и экспериментальные исследования, существенное воздействие оказывает именно однополярная составляющая импульса, а длинный фронт обратной полярности практически не влияет на систему $[3,11,12]$.

Униполярные импульсы большой электрической площади можно попытаться получить, используя явление самоиндуцированной прозрачности (СИП) [24,25]. При СИП передний фронт короткого импульса переводит среду из основного состояния в возбужденное, а на заднем фронте среда возвращает поглощенную энергию импульсу и переходит в основное невозбужденное состояние. В таком случае излучение распространяется в среде практически без потерь. При этом может происходить сильное изменение формы импульсов.

Двухкаскадный компрессор одноцикловых импульсов на основе явления СИП был рассмотрен в работе [26]. В ней исходный биполярный одноцикловый импульс, состоящий из двух полуволн противоположной полярности, испытывал следующую трансформацию. Однополярные полуволны сокращали свою длительность и притягивались друг к другу. В результате сжатия и притяжения полуволн одноциклового импульса длительность одноциклового импульса сокращалась в несколько раз. Используя несколько сред с кратными частотами переходов, можно сократить длительность исходного импульса от нескольких фемтосекунд до нескольких аттосекунд [26].

Однако получаемый таким образом импульс остается биполярным. В данной работе мы покажем, что существует другой сценарий изменения формы одноциклового импульса при распространении в режиме СИП. 
Однополярные полуволны импульса будут не сближаться, a, наоборот, отставать друг от друга. На такую возможность указывает пример численного решения задачи распространения малоциклового импульса в плотной резонансной среде [27]. Падавший на среду импульс разбивался на несколько подимпульсов. Первый и основной импульс содержал несколько циклов. За ним следовала пара разнесенных в пространстве униполярных импульсов с противоположной полярностью. Два импульса с противоположными полярностями были получены при расчетах отражения малоциклового импульса от среды с квадратичной или кубичной нелинейностью [22]. В случае диссипативных солитонов СИП аналогичное решение наблюдалось в работах $[28,29]$.

Вопрос о возможных применениях резонансной среды для разнесения во времени двух униполярных импульсов не изучался и будет рассмотрен в настоящей работе. Будет сделано сравнение электрической площади отдельных униполярных компонент с величинами электрической площади, необходимой для возбуждения простейших квантовых систем.

Напомним, что в одномерных задачах распространения света в средах с диссипацией действует правило сохранения электрической площади импульса [7,30,31]. Поэтому, если отсутствует значительное отражение от границ сред излучения, которое может иметь униполярный характер, то из исходно биполярного импульса невозможно получить униполярное излучение. Площадь прошедшего среду излучения должна быть равна нулю.

\section{Масштаб электрической площади импульса квантовых систем}

В недавней работе [32] введена величина „атомной меры электрической площади“, которая задает величину электрической площади униполярного импульса, необходимую для опустошения основного состояния квантовой системы. Выражение имеет универсальный вид:

$$
S_{0}=\frac{2 \hbar}{a q} \text {. }
$$

В него входят заряд $q$, $\hbar-$ приведенная постоянная Планка и $a-$ характерный размер системы.

Выражение (1) применимо к различным системам. В силу соотношения неопределенностей Гейзенберга в системе с размером $\sim a$ импульс имеет величину порядка $\hbar / a$ [33]. С другой стороны, электрическая площадь импульса совпадает с изменением под действием среднего квантово-механического значения импульса, отнесенным к единичному электрическому заряду системы [34]. Таким образом, атомная мера электрической площади импульса равна характерному квантово-механическому импульсу „свободной“ системы.

Для атома водорода (1) $a=a_{0}$, где $a_{0}=0.5 \cdot 10^{-8} \mathrm{~cm} \quad$ - радиус первой боровской орбиты атома водорода. Тогда $S_{0}=\frac{2 \hbar}{a_{0} q}=$
$=8.78 \cdot 10^{-10} \mathrm{erg} \cdot \mathrm{s} / \mathrm{cm} \cdot \mathrm{ESU}$. Это значение электрической площади служит „атомной мерой“ электрической площади и задает масштаб электрической площади в процессе взаимодействия униполярного импульса c атомной системой. Если электрическая площадь импульса больше этой величины, такой импульс способен изменить состояние системы и в этом масштабе обладает большой электрической площадью.

Для водородоподобных образований в твердых телах, колебаний и вращений молекул, электронов в проводящих наночастицах характерные размеры $a$ на порядки больше и соответственно значения „меры“ меньше.

\section{Коэффициент преобразования биполярного излучения в униполярное}

Отметим еще раз, что в одномерных задачах распространения света в средах с диссипацией правило сохранения электрической площади не позволяет получить униполярное излучение из исходно биполярного импульса, если отсутствует значительное отражение от границ.

Однако в рассматриваемой нами задаче преобразования одноциклового импульса в два „изолированных“ (удаленных на расстояние, значительно превышающее их протяженность) униполярных импульса с противоположными полярностями уместно поставить вопрос о том, как изменится величина площади положительной/отрицательной составляющей биполярного импульса при преобразовании в разнесенные импульсы. Для этого предлагается ввести коэффициент преобразования $U$, равный отношению электрической площади $E_{\text {out }}$ одного из униполярных импульсов к половине электрической площади модуля напряженности поля $E_{i n}$ в биполярном импульсе:

$$
U=\frac{\left|\int E_{\text {out }} d t\right|}{1 / 2 \int\left|E_{\text {in }}\right| d t} .
$$

Данный коэффициент может быть полезен при оценке эффективности преобразования малоцикловых импульсов в „уединенные“ однополярные.

\section{Моделирование получения „уединенных“ аттосекундных импульсов большой электрической площади}

Для теоретического описания распространения коротких импульсов в резонансной среде применялась система уравнений Максвелла-Блоха, описывающая эволюцию недиагональных элементов матрицы плотности двухуровневой среды, разности населенности уровней и электрического поля. Данная система уравнений имеет 
вид [26]:

$$
\begin{aligned}
\frac{\partial \rho_{12}(z, t)}{\partial t}= & -\frac{\rho_{12}(z, t)}{T_{2}}+i \omega_{0} \rho_{12}(z, t) \\
& -\frac{i}{\hbar} d_{12} E(z, t) n(z, t), \\
\frac{\partial n(z, t)}{\partial t}= & -\frac{n(z, t)-n_{0}(z)}{T_{1}} \\
& +\frac{4}{\hbar} d_{12} E(z, t) \operatorname{Im} \rho_{12}(z, t), \\
P(z, t)= & 2 N_{0} d_{12} \operatorname{Re} \rho_{12}(z, t), \\
\frac{\partial^{2} E(z, t)}{\partial z^{2}}- & \frac{1}{c^{2}} \frac{E(z, t)}{\partial t^{2}}=\frac{4 \pi}{c^{2}} \frac{\partial^{2} P(z, t)}{\partial t^{2}} .
\end{aligned}
$$

Здесь $\rho_{12}-$ недиагональный элемент матрицы плотности, $n \equiv \rho_{11}-\rho_{22}-$ разность населенностей между основным и возбужденным состояниями двухуровневой системы, $P$ - поляризация среды, $N_{0}-$ концентрация активных центров, $E-$ напряженность электрического поля с фиксированной линейной поляризацией, $c-$ скорость света в вакууме, $\omega_{0}$ - частота резонансного перехода среды $\left(\lambda_{0}=2 \pi c / \omega_{0}\right.$ - длина волны резонансного перехода), $n_{0}-$ равновесная разность населенностей $\left(n_{0}=1\right.$ для поглощающей среды). Подробнее о применимости данной модели для данной задачи cM. [26].

В настоящей работе уравнения для матрицы плотности (2) и (3) решались численно с использованием метода Рунге-Кутта, волновое уравнение (5) - методом конечных разностей. Падающий импульс имеет форму

$$
E(t)=E_{0} e^{-\frac{(t-z / c)^{2}}{\tau_{p}^{2}}} \sin \left(\omega\left(t-\frac{z}{c}\right)\right)
$$

где $E_{0}-$ амплитуда импульса, $\tau_{p}-$ длительность импульса, а $\omega-$ центральная частота. В дальнейших расчетах центральная частота импульса равна частоте перехода среды, $\omega=\omega_{0}$. Параметры, использованные в приводимом примере расчетов, приведены в таблице. Подобные значения параметров среды можно реализовать в различных наноструктурах и полупроводниковых материалах [26,35-37]. Импульсы с использованными в расчетах параметрами в настоящее время можно получить экспериментально [38].

Результаты численного моделирования приведены на рис. 1. Одноцикловый импульс в форме (6), содержащий две униполярные полуволны противоположной полярности, входит в резонансную среду, границы которой показаны вертикальными линиями на рис. 1. Эти полуволны распространяются в среде с разной скоростью, что приводит к их разбеганию. В результате на выходе из среды образуется пара разнесенных во времени униполярных импульсов противоположной полярности. Зависимость от времени напряженности электрического поля импульса на входе в среду и на выходе из среды показана на рис. 2.
Параметры, использованные в приводимом примере

\begin{tabular}{l|c}
\hline \multicolumn{1}{c|}{ Параметр } & Значение \\
\hline Толщина среды, $\mu \mathrm{m}$ & 4.5 \\
Концентрация $N_{0}, \mathrm{~cm}^{-3}$ & $8.5 \cdot 10^{22}$ \\
Дип. момент $d_{12}, \mathrm{D}$ & 5 \\
Время релаксации $T_{1}, \mathrm{ps}$ & 10 \\
Время релаксации $T_{2}, \mathrm{ps}$ & 10 \\
Центральная длина волны перехода $\lambda_{0}, \mathrm{~nm}$ & 700 \\
Частота перехода $\omega_{0}, \mathrm{~s}^{-1}$ & $2.69 \cdot 10^{15}$ \\
Амплитуда входного импульса $E_{0}, \mathrm{ESU}$ & $1.8 \cdot 10^{6}$ \\
Длительность входного импульса, $\tau_{p}, \mathrm{fs}$ & 2.61
\end{tabular}

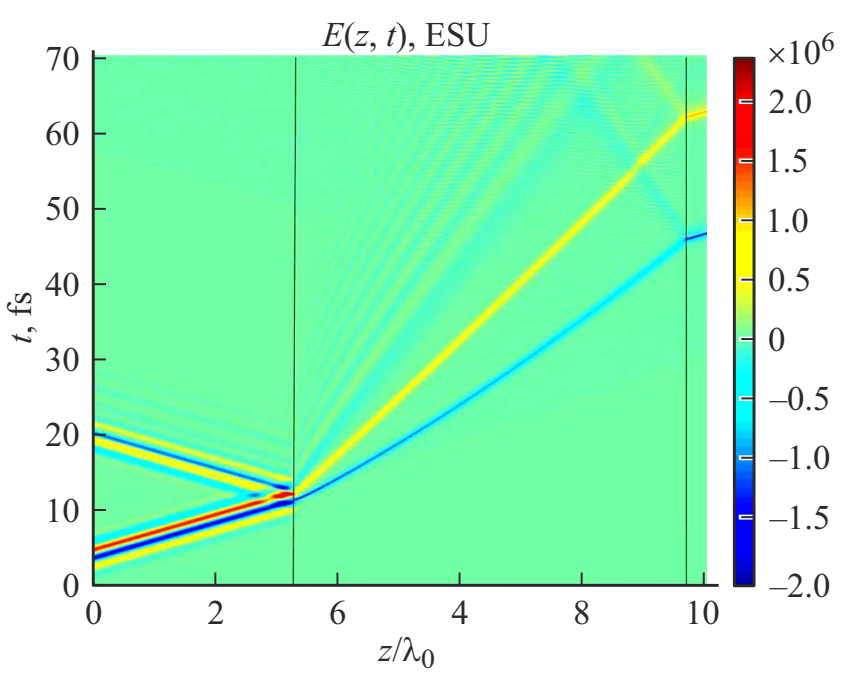

Рис. 1. Эволюция одноциклового импульса при распространени в двухуровневой резонансной среде, границы которой обозначены вертикальными линиями. По обе стороны от среды расположен вакуум.

Расчеты показали, что на расстояние между импульсами на выходе из поглощающей среды будет влиять концентрация частиц. Зависимость расстояния между импульсами от концентрации дана на рис. 3. Она имеет три характерных участка (выделены цветом). На первом участке, когда концентрации менее $3 \cdot 10^{22} \mathrm{~cm}^{-3}$, расстояние между импульсами равно расстоянию между полуволнами в исходном импульсе. Затем идет участок концентраций до $5.5 \cdot 10^{22} \mathrm{~cm}^{-3}$, где наблюдается компрессия импульса, которая было описана в работе [26]. После участка с компрессией расположена область, где происходит „расталкивание“ униполярных импульсов.

При значении концентрации $9 \cdot 10^{22} \mathrm{~cm}^{-3}$ и более второй импульс начинает менять свою форму, его длительность увеличивается, и появляются осцилляции, (рис. 4).

Интересна зависимость задержки между импульсами от времени $T_{2}$, которая дана на рис. 5. Из рисунка видно, что уменьшение времени $T_{2}$ сначала не влияет на задержку между импульсами, а затем приводит к ее 
быстрому уменьшению. Подобное поведение понятно, если считать, что первый импульс оставляет после себя среду в таком состоянии, которое замедляет движение второго импульса. При крайне малом значении времени $T_{2}$ воздействие первого импульса на второй пропадает, и поэтому импульсы не „расталкиваются““

Из приведенного примера следует, что при длине среды $6.4 \mu \mathrm{m}$ временной интервал между двумя импульсами $20 \mathrm{fs}$. Увеличение длины среды даст увеличение расстояния между импульсами, однако одновременно может происходить и разрушение такого режима распространения.

Как уже отмечалось, два разнесенных во времени униполярных импульса обладают способностью селективно возбуждать квантовые системы. Селективность воздействия определяется величиной задержки между ними $[11,12]$. При надлежащем выборе задержки второй импульс может усиливать воздействие первого. Это актуально, например, в задачах получения сверхизлучения „остановленной поляризации“ [39]. Величину задержки можно регулировать длиной среды. Изменение толщины
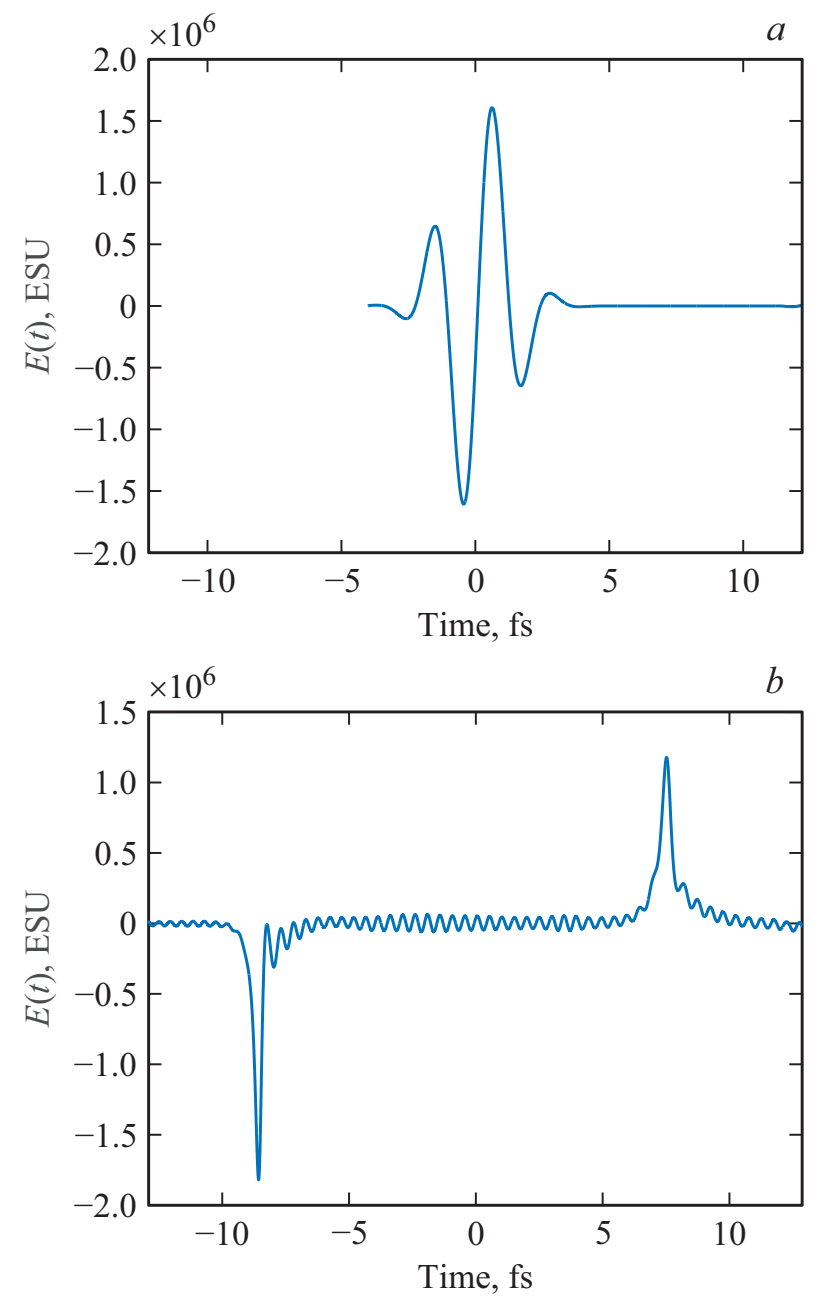

Рис. 2. Зависимости напряженности электрического поля $E(t)$ от времени на входе в среду $(a)$ и на выходе $(b)$ из неё.

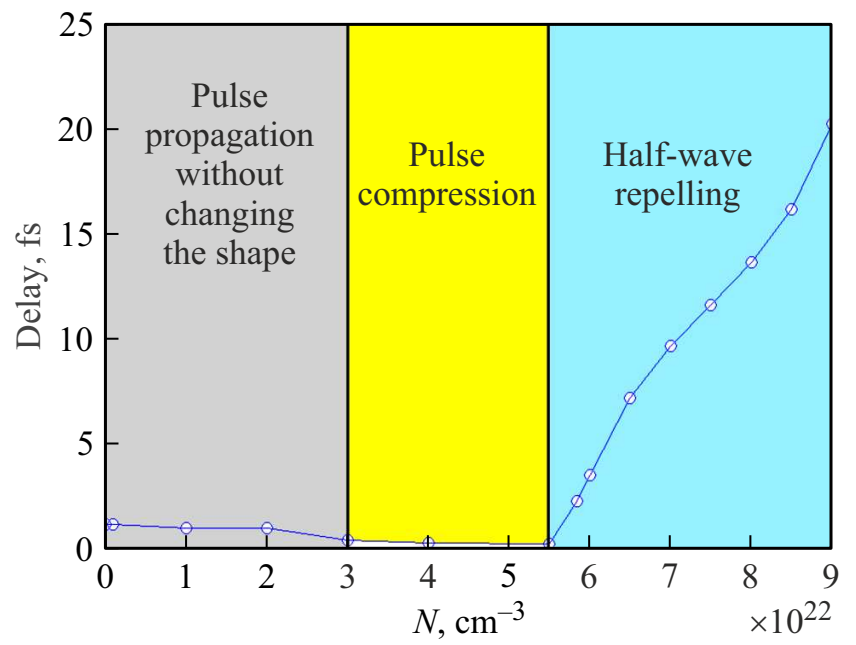

Рис. 3. Зависимость задержки между импульсами от концентрации $N_{0}$. Остальные параметры те же, что и на рис. 1. На рисунке обозначены области параметров, при которых импульс распространяется без изменения формы, зона компрессии входного импульса и область, где расстояние между униполярными волнами растет.

можно создать при наклоне тонкой пластинки относительно направления распространения пучка.

Отметим, что площадь импульса на рис. $2, b$ равна $8.7 \cdot 10^{-10} \mathrm{erg} \cdot \mathrm{s} / \mathrm{cm} \cdot \mathrm{ESU}$. Половина площади модуля напряженности поля падающего импульса на рис. $2, a$ равна $1.7 \cdot 10^{-9} \mathrm{erg} \cdot \mathrm{s} / \mathrm{cm} \cdot \mathrm{ESU}$. В приведенном примере коэффициент преобразования $U=0.5$.

\section{Подавление одной „уединенной“ компоненты при столкновении импульсов}

Если в среде формируется излучение в виде двух импульсов противоположной полярности, как на рис. $2, b$, то можно попытаться выделить один из них, направив навстречу короткий биполярный импульс. Ниже дан пример, который показывает такую возможность. Результаты соответствующего расчета даны на рис. 6. Расчет выполнен для среды длиной $3.8 \mu \mathrm{m}$, концентрации $N_{0}=7 \cdot 10^{22} \mathrm{~cm}^{-3}$ и времени релаксации $T_{2}=10^{-13} \mathrm{~s}$. Остальные параметры те же, что при расчете рис. 1.

В приведенном примере рис. $6, b$ встречный импульс имел меньшую амплитуду и ее знак был противоположен амплитуде исходного импульса. При смене знака амплитуды (рис. 6,c) оба коротких униполярных всплеска пропадали.

Сценарий столкновения, ведущий к уменьшению амплитуды второго униполярного всплеска и увеличению его длительности, показан на графиках поведения поля и разности населенностей в зоне столкновения (рис. 7).

Из рис. 7 видно, что направленный навстречу импульс также распадается на два униполярных импульса. В си- 


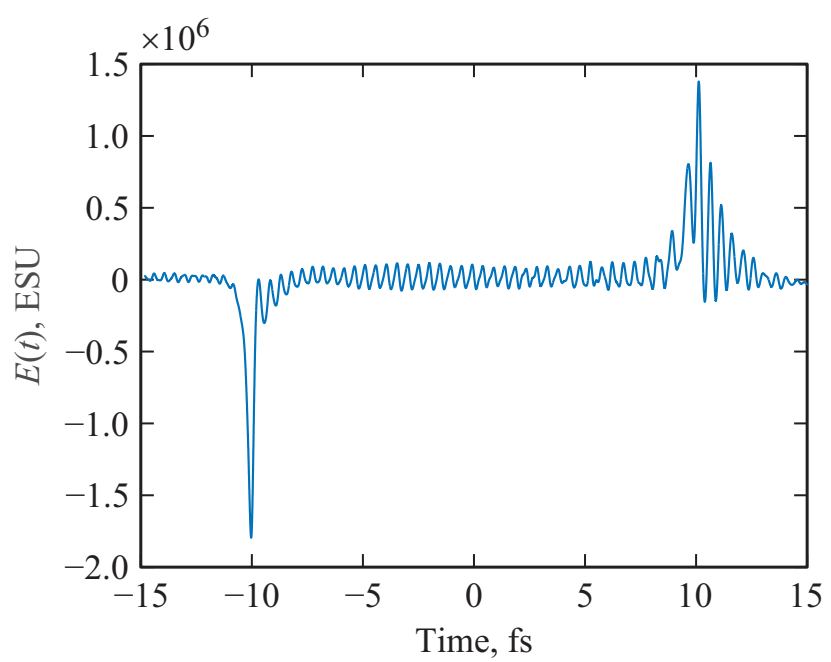

Рис. 4. Напряженность поля $E(t)$ на выходе из среды при $N_{0}=9 \cdot 10^{22} \mathrm{~cm}^{-3}$.

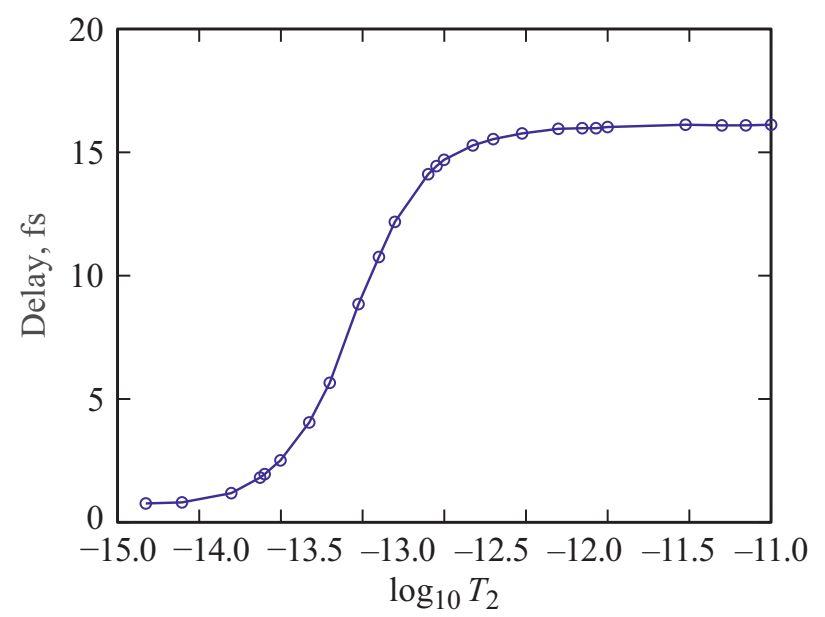

Рис. 5. Зависимость задержки между импульсами от времени релаксации $T_{2}$. По оси абсцисс отложен десятичный логарифм от $T_{2}$, выраженного в секундах. Остальные параметры те же, что и на рис. 1.

туации, когда удается выделить один импульс, первая компонента исходного импульса и встречного имеют противоположные полярности. Амплитуда встречного импульса меньше. Складываясь в области столкновения, поле встречного импульса незначительно ослабляет первый униполярный импульс и потом значительно ослабляет вторую униполярную волну. Это показано на рис. $6, b$. В ситуации, когда их амплитуды имеют одинаковый знак, соответственно ослабляется также первая волна, (рис. 6,c). Обратим внимание, что после столкновения первых частей встречных импульсов формируется мелкомасштабная структура разности населенностей (рис. 7, $b, c)$, сквозь которую должна пройти запаздывающая волна. Эта структура является источником излучения. Поэтому мы наблюдаем быструю модуляцию поля в ситуациях на рис. $6, b, c$.

\section{Обсуждение и выводы}

В работе теоретически продемонстрирована возможность получения субцикловых униполярных импульсов с большой электрической площадью на основе явления СИП при распространении одноциклового биполярного импульса в двухуровневой резонансно поглощающей среде. Показано, что при распространении исходного импульса его униполярные компоненты с противоположной полярностью движутся в среде с разной скоростью, что приводит к формированию на выходе из среды двух „уединенных“ униполярных импульсов с противоположной полярностью, разнесенных во времени. Длительность получаемых субцикловых импульсов составляет порядка 390 as. Электрическая площадь импульсов совпадает с величиной ,атомной меры электрической площади“ для атома водорода, и такой импульс обладает большой электрической площадью.

Показано, что можно сформировать субцикловый импульс с коротким по длительности всплеском большой полярности одной полярности и длинным фронтом противоположной полярности при столкновении двух биполярных импульсов.
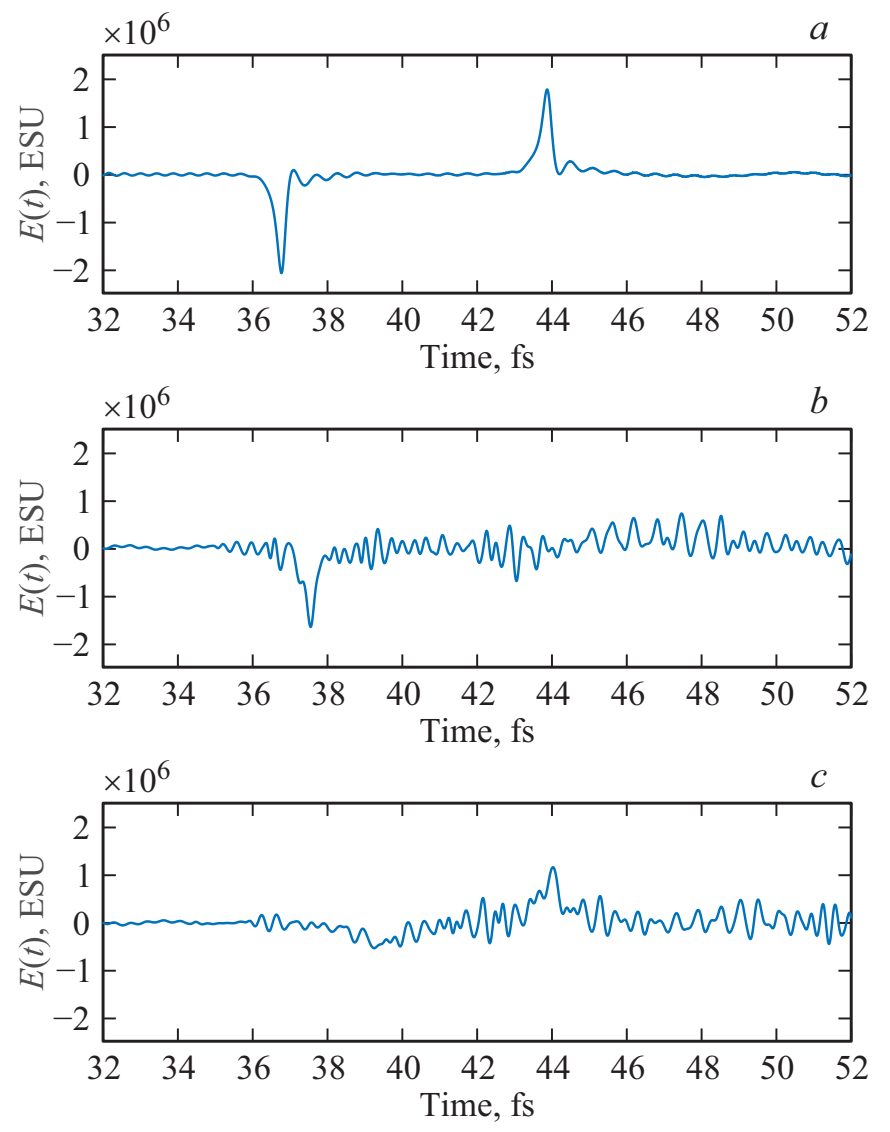

Рис. 6. Излучение на выходе из среды: $a-$ в отсутствие встречного импульса; $b-$ при наличии встречного импульса с амплитудой огибающей $E_{0}=-1.7 \cdot 10^{6} \mathrm{ESU} ; c-$ при амплитуде огибающей встречного импульса $E_{0}=1.7 \cdot 10^{6} \mathrm{ESU}$. 

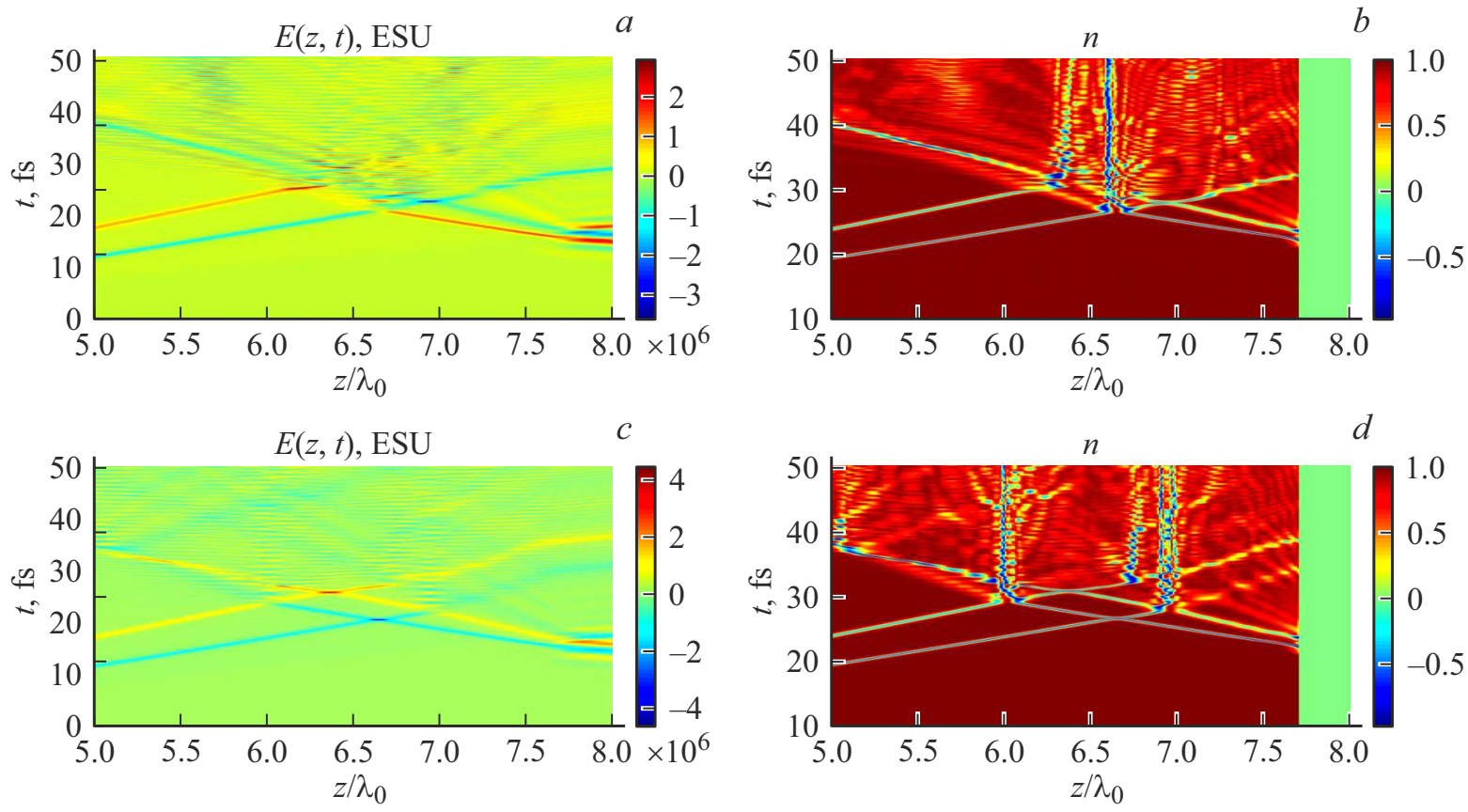

Рис. 7. Участки среды, где происходит столкновение импульсов. Зависимость напряженности поля $E$ (a,c) и разности населенности $n(b, d)$ в условиях рис. $6, b(a, b)$ и $6, c(c, d)$.

Рассмотренные ситуации трансформации биполярного импульса могут рассматриваться как варианты получения униполярных импульсов с большой электрической площадью, необходимых для быстрого и эффективного управления квантовыми системами и ускорения заряженных частиц.

\section{Финансирование работы}

Работа выполнена при финансовой поддержке РФФИ в рамках научного проекта 20-32-70049.

\section{Информация о вкладе авторов}

Расчеты выполнены с использованием программ, модифицированных С.В. Федоровым.

\section{Конфликт интересов}

Авторы заявляют, что у них нет конфликта интересов.

\section{Список литературы}

[1] Krausz F., Ivanov M. // Rev. Mod. Phys. 2009. V. 81. P. 163.

[2] Calegari F., Sansone G., Stagira S., Vozzi C., Nisoli M. // J. Phys. B: Atomic, Molecular and Optical Physics. 2016. V. 49. N 6. P. 062001.

[3] Hassan M.T., Luu T.T., Moulet A., Raskazovskaya O., Zhokhov P., Garg M., Karpowicz N., Zheltikov A.M., Pervak V., Krausz F., Goulielmakis E. // Nature. 2016. V. 530. P. 66.
[4] Rossi G.M., Mainz R.E., Yang Y., Scheiba F., SilvaToledo M.A., Chia S.H., Keathley P.D., Fang S., Mücke O.D., Manzoni C., Cerullo G., Cirmi G., Kärtner F.X. // Nature Photonics. 2020. V. 14. N 10. P. 629-635.

[5] Shou Y., Hu R., Gong Z., Yu J., Chen J., Mourou G., Yan X., Ma W. // New J. Phys. 2021. V. 23. N 5. P. 053003.

[6] Biegert J., Calegari F., Dudovich N., Quéré F., Vrakking M. // J. Physics. B: Atomic, Molecular and Optical Physics. 2021. V. 54. N 7. P. 070201.

[7] Розанов Н.Н., Архипов Р.М., Архипов М.В. // УФН. Т. 188. C. 1347; Rosanov N.N., Arkhipov R.M., Arkhipov M.V. // Phys. Usp. 2018. V. 61. P. 1227.

[8] Архипов Р.М., Архипов М.В., Розанов Н.Н. // Квант. электрон. 2020. Т. 50. № 9. С. 801-815; Arkhipov R.M., Arkhipov M.V., Rosanov N.N. // Quant. Electron. 2020. V. 50. N 9. P. $801-815$.

[9] Розанов Н.Н. // Опт. спектр. 2018. Т. 124. С. 75; Rosanov N.N. // Opt. Spectrosc. 2018. V. 124. P. 72.

[10] Arkhipov R.M., Arkhipov M.V., Babushkin I., Demircan A., Morgner U., Rosanov N.N. // Opt. Lett. 2019. V. 44. N 5. P. 1202.

[11] Архипов Р.М., Архипов М.В, Пахомов А.В., Розанов Н.Н. // Опт. и спектр. 2020. Т. 128. Вып. 1. C. 106-109; Arkhipov R.M., Arkhipov M.V., Pakhomov A.V., Rosanov N.N // Opt. Spectrosc. 2020. V. 128. N 1. P. 102-105.

[12] Arkhipov R., Pakhomov A., Arkhipov M., Demircan A., Morgner U., Rosanov N., Babushkin I. // Optics Express. 2020. V. 28. № 11. P. 17020-17034.

[13] Arkhipov R., Pakhomov A., Arkhipov M. Babushkin I. Demircan A., Morgner U., Rosanov N. // Scientific Reports. 2021. V. 11. Art. nr 1961.

[14] Архипов Р.М. // Письма в ЖЭТФ. 2021. Т. 113. № 6. С. 656; Arkhipov R.M. // JETP Lett. 2021. V. 113. N 10. P. 611. 
[15] Розанов Н.Н., Высотина Н.В. // ЖЭТФ. 2020. Т. 157. № 1. C. 63-66; Rosanov N.N., Vysotina N.V. // JETP. 2020. V. 130. N 1. P. 52-55.

[16] Aleksandrov I.A., Tumakov D.A., Kudlis A., Shabaev V.M., Rosanov N.N. // Phys. Rev. A. 2020. V. 102. P. 023102.

[17] Розанов Н.Н. // Письма в ЖЭТФ. 2021. Т. 113. № 3. С. 157; Rosanov N.N. // JETP Lett. 2021. V. 113. N 3. P. 145.

[18] Архипов Р.М., Архипов М.В., Розанов Н.Н. // Письма в ЖЭТФ. 2020. Т. 111. C. 586; Arkhipov R.M., Arkhipov M.V., Rosanov N.N. // JETP. Lett. 2020. V. 111. P. 484.

[19] Wu H.-C., Meyer-ter-Vehn J. // Nature Photon. 2012. V. 6. P. 304.

[20] Xu J., Shen B., Zhang X., Shi Y., Ji L., Zhang L., Xu T., Wang W., Zhao X., Xu Z. // Sci. Rep. 2018. V. 8. P. 2669.

[21] Feng L., Mccain J., Qiao Y. // Laser Phys. 2021. V. 31. P. 055301.

[22] Kozlov V.V., Rosanov N.N., De Angelis C., Wabnitz S. // Physical Review A. 2011. V. 84. N 2. P. 023818.

[23] Fülöp J.A., Tzortzakis S., Kampfrath T. // Adv. Opt. Mater. 2020. V. 8. P. 1900681.

[24] McCall S.L., Hahn E.L. // Phys. Rev. 1969. V. 183. P. 457.

[25] Аллен Л., Эберли Дж. Оптический резонанс и двухуровневые атомы. М.: Мир, 1978; Allen L., Eberly J.H. Optical Resonance and Two-level Atoms. NY: Wiley, 1975.

[26] Arkhipov R., Arkhipov M., Demircan A., Morgner U., Babushkin I., Rosanov N. // Opt. Express. 2021. V. 29. P. 10134.

[27] Kalosha V.P., Herrmann J. // Phys. Rev. Lett. 1999. V. 83. P. 544.

[28] Розанов Н.Н., Семенов В.Е., Высотина Н.В. // Квант. электрон. 2008. Т. 38. № 2. С. 137; Rosanov N.N., Semenov V.E., Vysotina N.V. // Quantum Electron. 2008. V. 38. P. 137.

[29] Розанов Н.Н. Диссипативные оптические солитоны. От микро- к нано- и атто-. М.: Физматлит, 2011.

[30] Розанов Н.Н. // Опт. и спектроск. 2009. Т. 107. № 5. С. 761; Rosanov N.N. // Opt. and Spectrosc. 2009. V. 107. N 5. P. 721.

[31] Arkhipov R., Arkhipov M., Babushkin I., Pakhomov A., Rosanov N. // J. Opt. Soc. Amer. B. 2021. V. 38. N 6. P. $2004-$ 2011.

[32] Архипов Р.М., Архипов М.В., Пахомов А.В., Розанов Н.Н. // Письма в ЖЭТФ. 2021. Т. 114. № 3, C. 156-159; Arkhipov R.M., Arkhipov M.V., Pakhomov A.V., Rosanov N.N. // JETP Lett. 2021. V. 114. N 3, P. 1-3.

[33] Ландау Л.Д., Лифииц Е.М. Квантовая механика. Нерелятивистская теория. М.: Наука, 1989; Landau L.D., Lifshitz E.M. Quantum Mechanics: Non-relativistic Theory. Oxford: Pergamon Press, 1977.

[34] Розанов Н.Н. // Опт. спектр. 2018. Т. 125. С. 818; Rosanov N.N. // Opt. Spectrosc. 2018. V. 125. P. 1012.

[35] Ryou J., Kim Y.S, Santosh K.C., Cho K. // Sci. Rep. 2016. V. 6(1). P. 29184.

[36] Yuan L., Hu W., Zhang H., Chen L., Wang Q. // Front. Bioeng. Biotechnol. 2020. V. 8. P. 21.

[37] Wu P.C., Kim T.H., Suvorova A., Giangregorio M., Saunders M., Bruno G., Brown A.S., Losurdo M. // Small. 2011. V. 7. P. $751-756$.

[38] Brahms C., Belli F., Travers J.C. // Phys. Rev. Research. 2020. V. 2. N 4. P. 043037.
[39] Архипов Р.М., Архипов М.В., Пахомов А.В., Жукова М.О., Цыпкин А.Н., Розанов Н.Н. // Письма в ЖЭТФ. 2021. T. 113. № 4. C. 237-247; Arkhipov R.M., Arkhipov M.V., Pakhomov A.V., Zhukova M.O., Tsypkin A.N., Rosanov N.N. // JETP Lett. 2021. V. 113. N 4. P. 242-251. 